\title{
The optimal timing of antihypertensive medication administration for morning hypertension in patients with cerebral infarction
}

\author{
Naohisa Hosomi ${ }^{1,2}$, Yoshimasa Sueda ${ }^{1}$, Hisashi Masugata ${ }^{3}$, Hiroaki Dobashi ${ }^{4}$, Koji Murao ${ }^{4}$, Masaki Ueno ${ }^{5}$, \\ Takanori Miki ${ }^{6}$, Masakazu Kohno ${ }^{2}$, Akira Nishiyama ${ }^{7}$ and Masayasu Matsumoto ${ }^{1}$
}

Morning hypertension is an independent risk factor for cardiovascular diseases, particularly stroke. However, the optimal time at which to take antihypertensive medication to treat morning hypertension remains unclear. We prospectively enrolled elderly patients (over 65 years old) with morning hypertension who had suffered an ischemic stroke (or strokes). Additional treatments (one of six arms) were randomly administered for 10 weeks in the morning, in the evening or at bedtime ( $n=15$ for each time point/medication). The patients measured their blood pressure and heart rate at home for 14 days prior to the intervention and for the final 14 days, and recorded the data in a blood pressure diary. The patients' urinary albumin/creatinine ratios were evaluated before and after the 10-week intervention. A total of 270 patients were enrolled in this study (mean age: $75.6 \pm$ 5.8 years; female/male ratio: 125/145). Their morning and evening systolic blood pressures were significantly decreased after following any of the study medication dosing schedules $(P<0.001)$. However, the reductions in the differences between the morning and evening systolic blood pressures were significant only when the medication was taken in the evening or at bedtime $(\boldsymbol{P}<0.001$ with repeated measures analysis of variance). Furthermore, the recovery rate from morning hypertension was also higher when the medication was taken in the evening $(40.0 \%)$ or at bedtime $(45.6 \%)$, rather than in the morning $(22.2 \%$; $P=0.003$ with the $\chi^{2}$-test). Antihypertensive medication taken in the evening or at bedtime is the most effective in treating morning hypertension when the patient adheres to the medication regimen.

Hypertension Research (2012) 35, 720-724; doi:10.1038/hr.2012.25; published online 1 March 2012

Keywords: antihypertensive medication; cerebrovascular disease; morning hypertension

\section{INTRODUCTION}

Hypertension is a major independent risk factor for cardiovascular diseases, especially stroke, and antihypertensive therapy is effective in preventing cardiovascular events. ${ }^{1-4}$ Blood pressure commonly exhibits a diurnal variation, peaking during the morning and then declining, to reach a minimum at approximately midnight. Similarly, there is a marked diurnal variation in the onset of cardiovascular events, with a peak incidence of myocardial infarction, sudden cardiac death, and ischemic and hemorrhagic strokes occurring in the morning (from 0600 hours to noon). ${ }^{5}$ Several prospective studies that evaluated self-measured home blood pressure have indicated that morning hypertension, which includes the morning surge, is an important risk factor for stroke and cardiovascular events. ${ }^{6}$
Antihypertensive medication is usually prescribed to be taken once in the morning to increase the likelihood of patient adherence. However, this medication schedule does not satisfactorily decrease morning hypertension. To date, several reports have evaluated the optimal time for taking antihypertensive medication to treat morning hypertension. ${ }^{7,8}$ However, those studies either compared morning medication schedules with evening schedules or morning schedules with bedtime schedules but did not compare all three schedules to evaluate their effectiveness at lowering morning hypertension. Therefore, it is still unknown whether taking medications in the evening or at bedtime is better than morning administration to treat morning hypertension. The purpose of this study was to define the optimal dosing schedule for antihypertensive medications to treat morning hypertension.

\footnotetext{
${ }^{1}$ Department of Clinical Neuroscience and Therapeutics, Graduate School of Biomedical Science, Hiroshima University, Hiroshima, Japan; ${ }^{2}$ Department of Cardiorenal and Cerebrovascular Medicine, Kagawa University School of Medicine, Kagawa, Japan; ${ }^{3}$ Department of Integrated Medicine, Kagawa University School of Medicine, Kagawa, Japan; ${ }^{4}$ Department of Internal Medicine, Kagawa University School of Medicine, Kagawa, Japan; ${ }^{5}$ Department of Pathology and Host Defence, Kagawa University School of Medicine, Kagawa, Japan; ${ }^{6}$ Department of Anatomy and Neurobiology, Kagawa University School of Medicine, Kagawa, Japan and ${ }^{7}$ Department of Pharmacology, Kagawa University School of Medicine, Kagawa, Japan

Correspondence: Dr N Hosomi, Department of Clinical Neuroscience and Therapeutics, Graduate School of Biomedical Science, Hiroshima University, 1-2-3, Kasumi, Minami-ku, Hiroshima, Hiroshima 734-8551, Japan.

E-mail: nhosomi@hiroshima-u.ac.jp

Received 2 December 2011; revised 26 December 2011; accepted 28 December 2011; published online 1 March 2012
} 


\section{METHODS}

\section{Study design and patients}

We prospectively enrolled elderly patients (over 65 years old) with morning hypertension who had a history of ischemic stroke (or strokes), could record their home blood pressure consistently and could visit the outpatient clinic at Kagawa University School of Medicine Hospital or Osaka Neurosurgical Hospital from April 2008 to December 2009. We excluded patients who had poor medication adherence ( $<95 \%$ of doses taken at the proper time). In addition, patients were also excluded if they were not good candidates to receive an additional antihypertensive medicine because of severe carotid stenosis (more than 75\% stenosis according to the North American Symptomatic Carotid Endarterectomy Trial criteria ${ }^{9}$ ), liver dysfunction (aspartate aminotransferase $\geqslant 100 \mathrm{IUl}^{-1}$ or alanine aminotransferase $\geqslant 100 \mathrm{IUl}^{-1}$ ), renal dysfunction (creatinine $\geqslant 1.5 \mathrm{mg} \mathrm{dl}^{-1}$ ) or an allergy to the medicine. All the patients who enrolled in this study provided their informed consent to participate. Our protocol was approved by the ethics review committee of the Kagawa University School of Medicine Hospital, Kagawa, Japan. The study protocol was registered on a clinical trials registration site (University Hospital Medical Information Network Clinical Trials Registry (UMIN-CTR: \#UMIN000001764)).

First, we evaluated the subjects' self-measured home blood pressure and heart rate for 14 days. The patients were asked to record these values in a blood pressure diary. Then each patient was randomly assigned to receive one of the interventional treatments (the calcium channel blocker amlodipine $5 \mathrm{mg}$ per day; the angiotensin-converting enzyme inhibitor perindopril $4 \mathrm{mg}$ per day; or the angiotensin II receptor blocker candesartan $8 \mathrm{mg}$ per day, olmesartan $20 \mathrm{mg}$ per day, valsartan $80 \mathrm{mg}$ per day or telmisartan $40 \mathrm{mg}$ per day) for 10 weeks. Patients were not administered a medication from the same class as one of their current antihypertensive drugs, and the interventional treatment was added to each patient's current antihypertensive medication(s) in the morning (after breakfast), in the evening (after dinner) or at bedtime ( $n=15$ for each time point/medication).

We also assessed the presence of diabetes mellitus and dyslipidemia. Diabetes mellitus was defined as a fasting blood glucose level $\geqslant 126 \mathrm{mg} \mathrm{dl}^{-1}$ or the use of any antidiabetes drugs. Dyslipidemia was defined as a low-density lipoprotein cholesterol level $\geqslant 140 \mathrm{mg} \mathrm{dl}^{-1}$, a triglyceride level $\geqslant 150 \mathrm{mg} \mathrm{dl}^{-1}$, a high-density lipoprotein cholesterol level $<40 \mathrm{mgdl}^{-1}$ or the use of any antihyperlipidemia drugs. We also recorded the class(es) of patients' original antihypertensive medication(s).

\section{Home blood pressure}

Home blood pressure was measured according to the Japanese Society of Hypertension guidelines for the self-monitoring of blood pressure at home. ${ }^{10}$ Home blood pressure was measured with an electrical sphygmomanometer in a standardized manner following $10 \mathrm{~min}$ of rest in a sitting position. The blood pressure was measured three times with more than 1 min between samplings in the morning (within $30 \mathrm{~min}$ of awakening and prior to doing anything else except going to the restroom) and in the evening (before dinner). The median value of these three blood pressure measurements was used in the subsequent analyses. The home blood pressure and heart rate values were collected for 14 days prior to the intervention and during the final 14 days of the observational period, beginning 8 weeks after starting the intervention. The mean home blood pressure and heart rate values from the 14 days of measurement were used for analysis.

The diagnosis of morning hypertension was based on the home blood pressures recorded for 14 days prior to the intervention; patients were determined to have morning hypertension when the average of their morning and evening systolic blood pressures was $\geqslant 135 \mathrm{~mm} \mathrm{Hg}$ and the difference between the morning and evening systolic blood pressure (morning systolic blood pressure-evening systolic blood pressure) was $\geqslant 15 \mathrm{~mm} \mathrm{Hg}$, as described elsewhere. $^{8}$

\section{Microalbuminuria}

The urinary albumin/creatinine ratio (UACR, $\mathrm{mg} \mathrm{g}^{-1}$ ) was evaluated using fasting morning spot-urine tests conducted prior to the intervention and 10 weeks after starting the intervention. Urinary albumin levels were measured with the immunoturbidimetric method, and urinary creatinine levels were measured with the enzymatic method. UACR was calculated as described elsewhere. ${ }^{11}$

\section{Statistical analysis}

The data are expressed as the means \pm s.d. for continuous variables and as frequencies and percentages for discrete variables. Univariate analyses were performed to evaluate the differences between groups with respect to baseline characteristics, current medication and UACR. The clinical characteristics of the groups were compared using a one-way analysis of variance (ANOVA, for continuous variables) or Fisher's exact test (for discrete variables). Changes between the morning and evening systolic blood pressures during the interventional treatment period and changes in the UACR were examined using repeated measures ANOVA. The reduction rates of them were calculated with the formula (pre-value-post-value)/pre-value. Then, the differences of reduction rates between the each dosing schedules were examined using a one-way ANOVA. The rates of recovery from morning hypertension were examined using the $\chi^{2}$-test. When the $P$-value was $<0.05$ by ANOVA, Turkey-Kramer's Honestly Significant Difference test or Bonferroni's correction for multiple comparisons was applied to evaluate whether the differences in each betweengroups were significant. The difference of systolic blood pressure, diastolic blood pressure, the level of difference between the morning and evening systolic blood pressure (morning systolic blood pressure-evening systolic blood pressure), and UACR between the pre- and postinterventional treatment periods were evaluated for each medication dosing schedule using a paired $t$-test. The statistical analyses were performed using JMP software version 9.0 or StatView version 5.0 for Macintosh (SAS, Cary, NC, USA). All the analyses were two-tailed, and a value of $P<0.05$ was considered statistically significant.

\section{RESULTS}

\section{Patient characteristics}

The subjects' clinical characteristics, morning and evening systolic blood pressures, diastolic blood pressures, heart rates and baseline UACRs grouped according to morning, evening and bedtime antihypertensive medication schedules $(n=90)$ are listed in Table 1 . There were no significant differences in clinical characteristics between the different medication schedule groups.

\section{The appropriate timing of antihypertensive medication to treat morning hypertension}

There were no significant differences in the morning and evening systolic blood pressures, the diastolic blood pressures and the heart rates before the interventions between the different medication schedule groups (Table 1). All of the studied dosing schedules of antihypertensive medications effectively reduced morning and evening systolic blood pressures $(P<0.001$ using two-way repeated measures ANOVA, Figure 1). There were no significant differences in the changes of the morning and evening systolic blood pressures among the groups on different dosing schedules $(P=0.29$ and 0.86 using twoway repeated measures ANOVA, respectively). The reduction rates of morning systolic blood pressure with the morning, evening or bedtime dosing schedule were $0.119 \pm 0.023,0.121 \pm 0.018$ or $0.126 \pm 0.025$, respectively ( $P=0.026$, using one-way ANOVA). It was significantly higher in the bedtime medication schedule than that in the morning medication schedule $(P=0.021)$. Moreover, the reduction rates of evening systolic blood pressure with either medication schedules were $0.088 \pm 0.041,0.059 \pm 0.059$ or $0.059 \pm 0.054$, respectively $(P<0.001$, using one-way ANOVA). It was significantly higher in the morning medication schedule than that in the evening and bedtime medication schedule ( $P=0.001$ and $P<0.001$, respectively). In contrast, the difference between the morning and evening systolic blood pressure (morning systolic blood pressure-evening systolic blood pressure) was reduced significantly with the evening and 
Table 1 Clinical characteristics of the study cohort

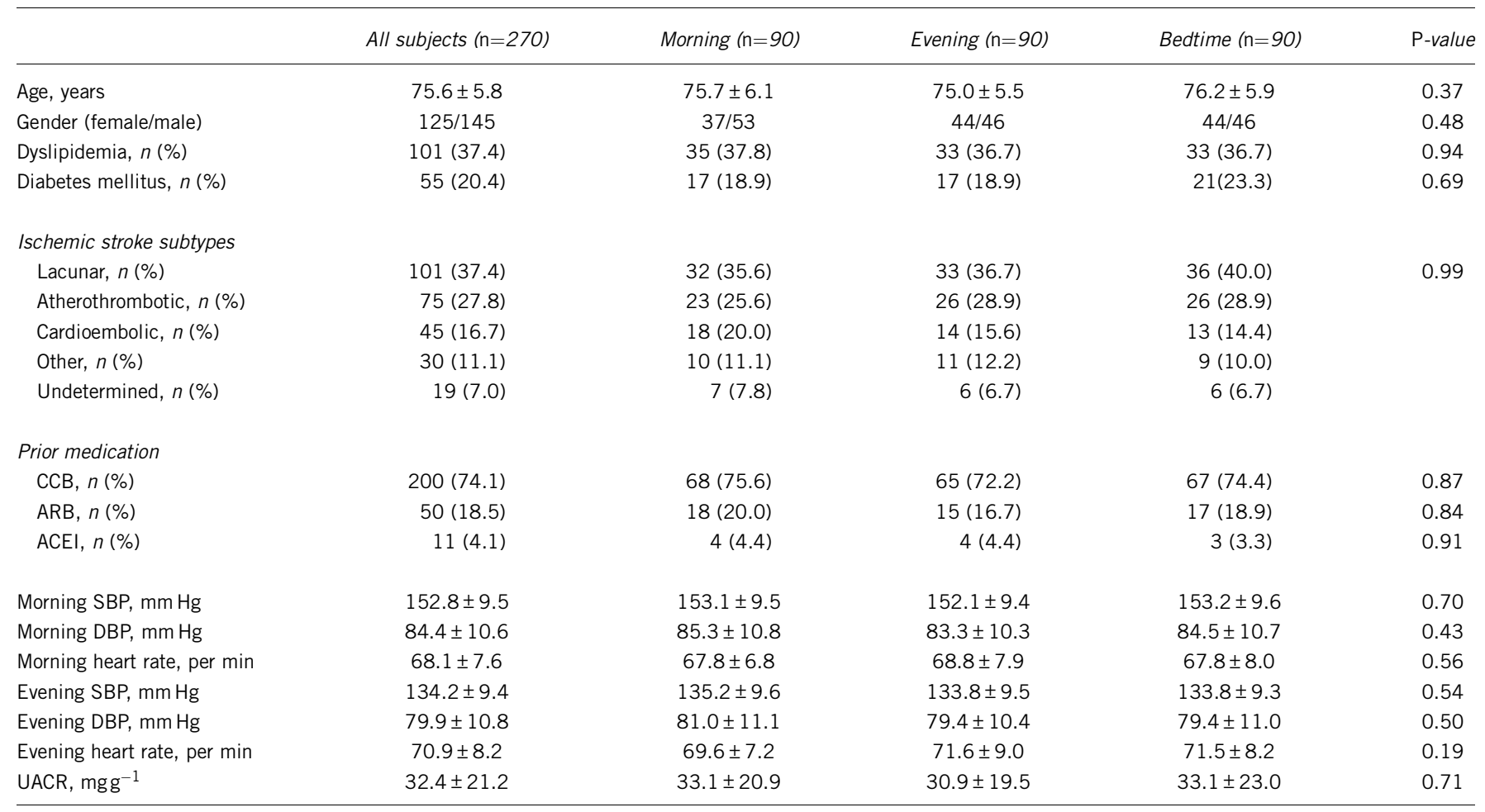

Abbreviations: ACEI, angiotensin-converting enzyme inhibitor; ARB, angiotensin II receptor blocker; CCB, calcium channel blocker; DBP, diastolic blood pressure; SBP, systolic blood pressure; UACR, urinary albumin/creatinine ratio.

bedtime dosing schedule groups $(P<0.001$, using two-way repeated measures ANOVA with Bonferroni's corrections, respectively, Figure 1). There were significant reductions in the changes of the difference between the morning and evening systolic blood pressure with the evening and bedtime medication schedules compared with the morning medication schedule $(P<0.001)$. The reduction rates of the difference between the morning and evening systolic blood pressure with the morning, evening or bedtime dosing schedule were $-0.345 \pm 1.05, \quad 0.387 \pm 0.908$ or $0.596 \pm 1.360$, respectively $(P<0.001$, using one-way ANOVA $)$. It was significantly higher in the evening and bedtime medication schedules than that in the morning medication schedule $(P<0.001)$. In addition, the proportion of patients who recovered from morning hypertension was evaluated with respect to the timing of antihypertensive medication administration (Figure 2). Recovery from morning hypertension was defined as the attainment of the following follow-up home blood pressure measurements for 14 days: morning and evening systolic blood pressures averaging $<135 \mathrm{~mm} \mathrm{Hg}$ and the difference between the morning and evening systolic blood pressure (morning systolic blood pressure-evening systolic blood pressure) averaging $<15 \mathrm{~mm} \mathrm{Hg}$. The rates of recovery from morning hypertension were higher among the patients who took their medications in the evening or at bedtime (40.0 and $45.6 \%$, respectively) compared with those on the morning schedule $\left(22.2 \% ; P=0.003\right.$ with the $\chi^{2}$-test).

\section{The appropriate arms of antihypertensive medication to treat morning hypertension}

There are significant differences in the reduction of the morning and evening systolic blood pressure among the medication arms $(P<0.001$, using two-way repeated measures ANOVA). Among the medication arms used in this study, amlodipine significantly reduced the morning and evening systolic blood pressure compared with the other arms $(P<0.001)$. However, there was no significant difference in the difference between the morning and evening systolic blood pressure among the medication arms $(P=0.85$, using two-way repeated measures ANOVA).

\section{Decreases in the UACR in each dosing schedule}

We evaluated the effects of the different dosing schedules of antihypertensive medications on lowering the UACR. There were no significant differences in the UACRs before the interventions between the different medication schedule groups (Table 1). All of the dosing schedules produced effective reductions in the UACR $(P<0.001$, using two-way repeated measures ANOVA, Figure 3). However, there were no significant differences in the amount of UACR reduction among the different schedules $(P=0.59$, using two-way repeated measures ANOVA). The reduction rates of UACR with the morning, evening or bedtime medication schedules were $0.148 \pm 0.068,0.167 \pm 0.169$ or $0.178 \pm 0.127$, respectively $(P=0.28$, using one-way ANOVA). In addition, there was no significant difference among the medication arms in the reduction of UACR $(P=0.14$, using two-way repeated measures ANOVA).

\section{DISCUSSION}

In this study, the optimal medication dosing schedule for the most effective treatment of morning hypertension was prospectively evaluated using six antihypertensive medications. Our results indicate that to reduce the difference between morning and evening blood pressures, antihypertensive medication should be taken in the evening or at bedtime and that evening or bedtime administration of these 

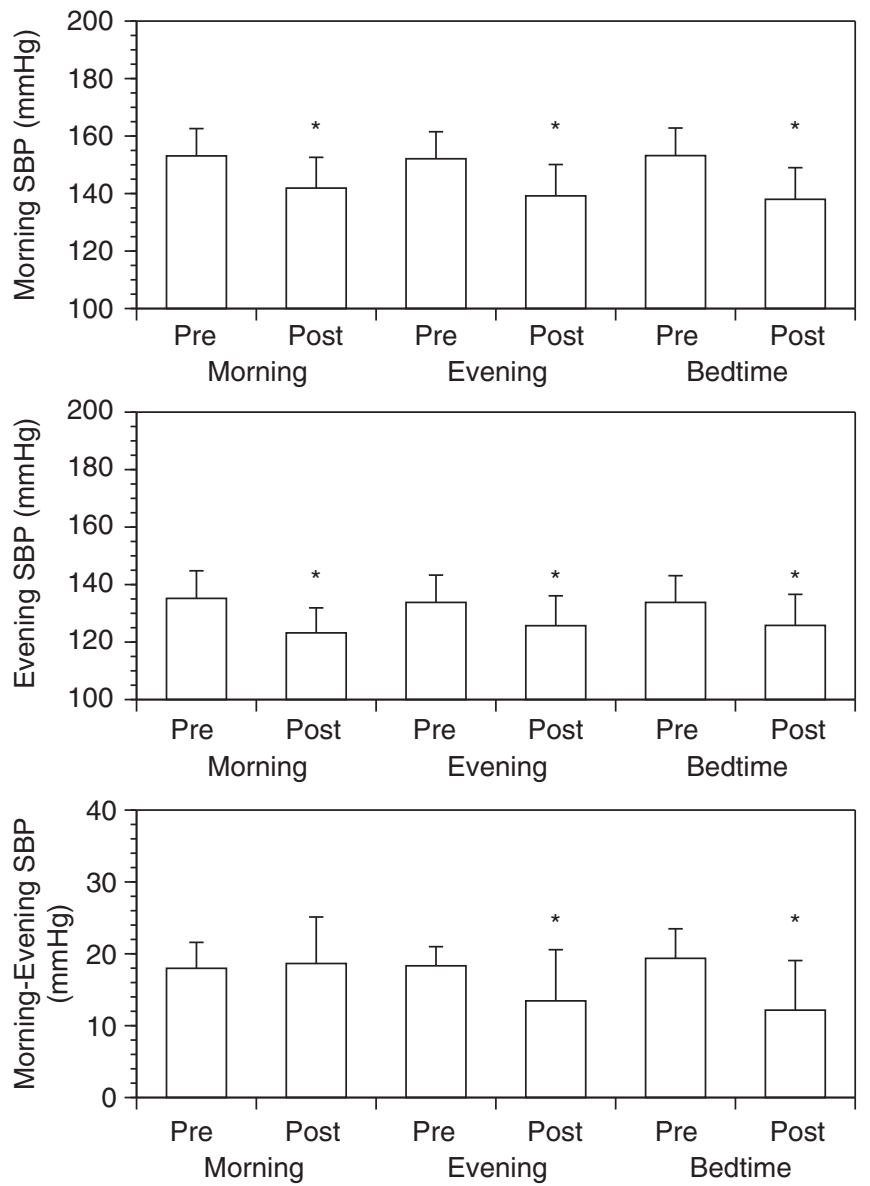

Figure 1 Differences in blood pressure reduction with different medication dosing schedules. Although there were no significant differences between the dosing schedules in terms of the levels of reduction of morning and evening SBPs, the difference between the morning and evening systolic blood pressure (morning-evening SBP) was significantly reduced in the groups that received evening or bedtime medications, but not in the group assigned to morning medications. SBP, systolic blood pressure. ${ }^{*} P<0.001$ compared with preintervention.

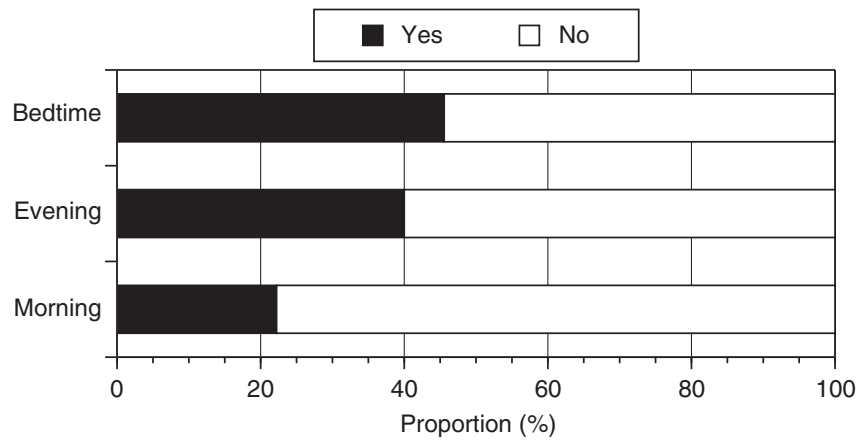

Figure 2 Recovery from morning hypertension according to the timing of medication administration. The recovery rate from morning hypertension was significantly higher following evening or bedtime dosing of medications compared with the morning dosing regimen. Recovery from morning hypertension was defined as the satisfaction of both of the following criteria: average morning and evening systolic blood pressure was $<135 \mathrm{~mm} \mathrm{Hg}$ and average difference between the morning and evening systolic blood pressure (morning systolic blood pressure-evening systolic blood pressure) was $<15 \mathrm{~mm} \mathrm{Hg}$. medications is more effective than morning administration at facilitating recovery from morning hypertension. However, there were no significant differences between the dosing schedules in the level of UACR reduction. Among the medication arms, amlodipine significantly reduced the morning and evening systolic blood pressure more than the other arms. However, there was no significant difference in the difference between morning and evening blood pressures among the medication arms.

Hypertension is known to be a major risk factor for cardiovascular events. In addition, it has been reported that antihypertensive medications strongly protect against cardiovascular diseases. ${ }^{12}$ Therefore, antihypertensive treatment is commonly used in the clinic. The next logical step is to increase the quality of blood pressure control in hypertensive patients. It has been reported that morning hypertension is a strong risk factor for stroke. ${ }^{6,13}$ The difference between the morning and evening self-measured blood pressures is an independent determinant of cardiac dysfunction. ${ }^{14}$ The potential cardiovascular advantages of controlling morning hypertension have not been fully demonstrated. On the other hand, there are several reports that showed the reduced cardiovascular risks with the bedtime antihypertensive medications compared with the morning medications. ${ }^{15-17}$ From this study, the bedtime antihypertensive medication was the most effective medication schedule reducing the morning systolic blood pressure and the difference between the morning and evening systolic blood pressure. Therefore, the bedtime antihypertensive medications could be the most effective timing to reduced cardiovascular risks controlling morning hypertension.

In this study, we evaluated the timing of the administration of antihypertensive medicine to control morning hypertension. We found that both evening and bedtime dosing of antihypertensive medication could effectively treat morning hypertension. Several studies support our results. ${ }^{7,8}$ However, those studies either compared morning medication schedules with evening schedules or morning schedules with bedtime schedules but did not compare all three schedules to evaluate their effectiveness at lowering morning hypertension. Therefore, this study is the first to compare the effectiveness of antihypertensive medication at lowering blood pressure in patients with morning hypertension, who adhered to dosing schedules that included morning, evening and bedtime administrations of medication.

Patients are usually instructed to take medication in the morning to increase their adherence to the regimen, unless a medication specifically should be taken at another time. For this study, we selected subjects who had exhibited high adherence $(\geqslant 95 \%)$ to a medication regimen. Therefore, there was high adherence (more than 90\%) to the dosing schedule, even among the patients who had been assigned to the evening or bedtime medication groups. However, there is a certain proportion of patients who show a low adherence to medication in general. ${ }^{18}$ Therefore, when it is necessary to treat a patient with morning hypertension who does not comply well with medication regimens, prioritizing adherence is important because evening or bedtime dosing schedules may decrease compliance.

We also evaluated differences in the levels of reduction of the UACR between the morning, evening and bedtime medication schedules. Our results show that there were no significant differences in the UACR reduction level between the medication schedules. In the Japan Morning Surge-Target Organ Protection (J-TOP) study, the UACR was reduced more with bedtime administration than with morning administration of the angiotensin II receptor blocker candesartan. ${ }^{8}$ However, in the J-TOP study, UACR reduction was evaluated over 6 months. In this study, we examined the effects of morning hypertension 


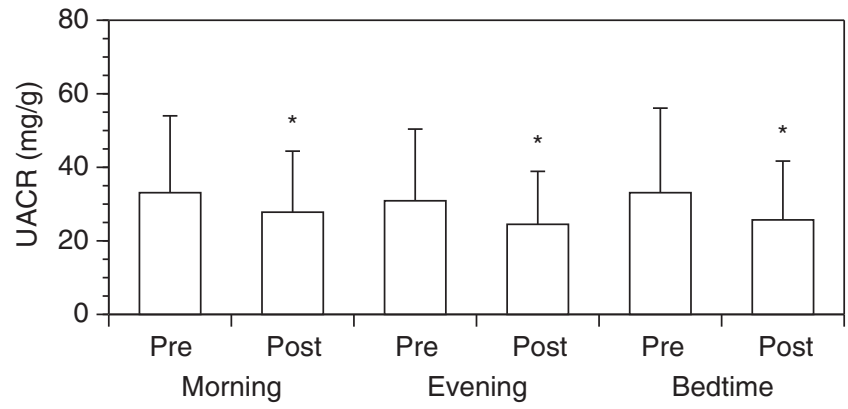

Figure 3 Effects of different dosing schedules on the urinary albumin/ creatinine ratio (UACR). Although there were significant reductions in the UACR with all the dosing schedules $(P<0.001$, for all comparisons), there were no significant differences in the levels of reduction of UACR between the timings of medication administration (analyzed with repeated measure ANOVA). ${ }^{*} P<0.001$ compared with preintervention.

treatment for 2 months. The effects of treating morning hypertension on organ protection remain controversial. More than 6 months may be necessary to acquire sufficient statistical power to observe a significant difference between evening and bedtime administration of antihypertensive medications in terms of organ protection. In addition, the effects of the appropriate treatment of morning hypertension on cardiovascular disease prevention have not yet been determined.

This study has several limitations. First, all of the patients had experienced both an ischemic stroke (or strokes) and morning hypertension. Therefore, the study population may not represent the general population of patients with morning hypertension. Second, as described above, this study was designed to find the best medication dosing schedule to treat morning hypertension over a 2-month period. This time frame may be too short to observe differences in organ protection, for example, UACR reduction, between the different dosing schedules. Third, we evaluated self-measured home blood pressures. To more accurately assess blood pressure control, it may be better to evaluate ambulatory blood pressure measurements in the same time, as both self-measured home blood pressures and ambulatory blood pressure measurements are known to have some complementary strong and weak points each other. Fourth, as the dosing schedule, we have only got the information of medication schedule as morning, evening or bedtime. To understand more detailed performances of each medication schedule, it may be better to know the exact timing at which the patients took medicine.

The optimal timing of antihypertensive medication administration to treat morning hypertension was evaluated in this study. Our results indicate that antihypertensive medication taken in the evening or at bedtime is the most effective in treating morning hypertension when the patient adheres to the medication regimen. We did not observe a difference in organ protection, that is, UACR reduction, between the different antihypertensive medication dosing schedules during the 10 -week intervention. Further studies are warranted to determine whether the appropriate treatment of morning hypertension could lead to organ protection or cardiovascular disease prevention.

\section{CONFLICT OF INTEREST}

The authors declare no conflict of interest.

\section{ACKNOWLEDGEMENTS}

We express our gratitude to Mayumi Ohguro and Yuka Kimura for their clerkship assistance. This study was partly supported by Grants in Aid for Scientific Research from the Ministry of Education, Culture, Sports \& Technology of Japan.

1 Tanizaki Y, Kiyohara Y, Kato I, Iwamoto H, Nakayama K, Shinohara N, Arima H, Tanaka K, Ibayashi S, Fujishima M. Incidence and risk factors for subtypes of cerebral infarction in a general population: the Hisayama study. Stroke 2000; 31: 2616-2622.

2 Lawes CM, Rodgers A, Bennett DA, Parag V, Suh I, Ueshima H, MacMahon S. Blood pressure and cardiovascular disease in the Asia Pacific region. J Hypertens 2003; 21: 707-716.

3 Lida M, Ueda K, Okayama A, Kodama K, Sawai K, Shibata S, Tanaka S, Keijnkai T, Horibe $\mathrm{H}$, Minowa M, Yanagawa H, Hashimoto T. Impact of elevated blood pressure on mortality from all causes, cardiovascular diseases, heart disease and stroke among Japanese: 14 year follow-up of randomly selected population from Japanese - Nippon data 80. J Hum Hypertens 2003; 17: 851-857.

4 Staessen JA, Wang JG, Thijs L. Cardiovascular prevention and blood pressure reduction: a quantitative overview updated until 1 March 2003. J Hypertens 2003; 21 : 1055-1076.

5 Muller JE, Tofler GH, Stone PH. Circadian variation and triggers of onset of acute cardiovascular disease. Circulation 1989; 79: 733-743.

6 Asayama K, Ohkubo T, Kikuya M, Obara T, Metoki H, Inoue R, Hara A, Hirose T, Hoshi H, Hashimoto J, Totsune K, Satoh H, Imai Y. Prediction of stroke by home 'morning' versus 'evening' blood pressure values: the Ohasama study. Hypertension 2006; 48: 737-743.

7 Hermida RC, Ayala DE, Fernandez JR, Calvo C. Comparison of the efficacy of morning versus evening administration of telmisartan in essential hypertension. Hypertension 2007; 50: 715-722.

8 Kario K, Hoshide S, Shimizu M, Yano Y, Eguchi K, Ishikawa J, Ishikawa S, Shimada K. Effect of dosing time of angiotensin II receptor blockade titrated by self-measured blood pressure recordings on cardiorenal protection in hypertensives: the Japan Morning Surge-Target Organ Protection (J-TOP) study. J Hypertens 2010; 28: 1574-1583.

9 North American Symptomatic Carotid Endarterectomy Trial Collaborators. Beneficial effect of carotid endarterectomy in symptomatic patients with high-grade carotid stenosis. N Eng/ J Med 1991; 325: 445-453.

10 Imai Y, Otsuka K, Kawano Y, Shimada K, Hayashi H, Tochikubo O, Miyakawa M, Fukiyama K. Japanese society of hypertension (JSH) guidelines for self-monitoring of blood pressure at home. Hypertens Res 2003; 26: 771-782.

11 Gerstein HC, Mann JF, Yi Q, Zinman B, Dinneen SF, Hoogwerf B, Halle JP, Young J, Rashkow A, Joyce C, Nawaz S, Yusuf S. Albuminuria and risk of cardiovascular events, death, and heart failure in diabetic and nondiabetic individuals. JAMA 2001; 286: 421-426.

12 Arima H, Chalmers J, Woodward M, Anderson C, Rodgers A, Davis S, Macmahon S, Neal B. Lower target blood pressures are safe and effective for the prevention of recurrent stroke: the PROGRESS trial. J Hypertens 2006; 24: 1201-1208.

13 Kario K, Pickering TG, Umeda Y, Hoshide S, Hoshide Y, Morinari M, Murata M, Kuroda T, Schwartz JE, Shimada K. Morning surge in blood pressure as a predictor of silent and clinical cerebrovascular disease in elderly hypertensives: a prospective study. Circulation 2003; 107: 1401-1406.

14 Matsui Y, Eguchi K, Shibasaki S, Shimizu M, Ishikawa J, Shimada K, Kario K. Association between the morning-evening difference in home blood pressure and cardiac damage in untreated hypertensive patients. J Hypertens 2009; 27: 712-720.

15 Hermida RC, Ayala DE, Mojon A, Fernandez JR. Influence of circadian time of hypertension treatment on cardiovascular risk: results of the MAPEC study. Chronobiol Int 2010; 27: 1629-1651.

16 Hermida RC, Ayala DE, Mojon A, Fernandez JR. Influence of time of day of blood pressure-lowering treatment on cardiovascular risk in hypertensive patients with type 2 diabetes. Diabetes Care 2011; 34: 1270-1276.

17 Hermida RC, Ayala DE, Mojon A, Fernandez JR. Bedtime dosing of antihypertensive medications reduces cardiovascular risk in CKD. J Am Soc Nephrol 2011; 22: 2313-2321.

18 Johnson MJ, Williams M, Marshall ES. Adherent and nonadherent medication-taking in elderly hypertensive patients. Clin Nurs Res 1999; 8: 318-335. 